\title{
Measuring $\Omega_{0}$ with higher-order quasar-galaxy correlations induced by weak lensing
}

\author{
B. Ménard ${ }^{1,2}$, M. Bartelmann ${ }^{1}$, and Y. Mellier ${ }^{2,3}$ \\ 1 Max-Planck-Institut für Astrophysik, PO Box 1317, 85741 Garching, Germany \\ 2 Institut d'Astrophysique de Paris, 98bis Bld. Arago, 75014 Paris, France \\ 3 LERMA, Observatoire de Paris, 61 avenue de l'Observatoire, 75014 Paris, France
}

Received 21 August 2002 / Accepted 20 May 2003

\begin{abstract}
Via the magnification bias, gravitational lensing by large-scale structures causes angular cross-correlations between distant quasars and foreground galaxies on angular scales of arcminutes and above. We investigate the three-point crosscorrelation between quasars and galaxy pairs measurable via the second moment of the galaxy counts around quasars and show that it reaches the level of a few per cent on angular scales near one arcminute. Combining two- and three-point correlations, a skewness parameter can be defined which is shown to be virtually independent of the shape and normalisation of the dark-matter power spectrum. If the galaxy bias is linear and deterministic, the skewness depends on the cosmic matter density parameter $\Omega_{0}$ only; otherwise, it can be used to probe the non-linearity and stochasticity of the bias. We finally estimate the signal-to-noise ratio of a skewness determination and find that a sample of about twenty thousand distant quasars e.g. from the Sloan Digital Sky Survey should suffice for a direct measurement of $\Omega_{0}$.
\end{abstract}

Key words. cosmology: gravitational lensing - cosmology: large-scale structure of Universe

\section{Introduction}

It is widely believed that structures and galaxies in the Universe formed from gravitational growth of Gaussian primordial mass density fluctuations dominated by dark matter. Direct support for this picture is provided by the recent weak lensing surveys of galaxies that measured the systematic distortion of faint background-galaxy images produced by the gravitational tidal field of intervening dark-matter inhomogeneities: the cosmic shear (Bacon et al. 2000, 2002; Hämmerle et al. 2002; Hoekstra et al. 2002; Kaiser et al. 2000; Maoli et al. 2001; Réfrégier et al. 2002; Rhodes et al. 2001; van Waerbeke et al. 2000, 2001, 2002; Wittman et al. 2000). The shape of the cosmic shear signal as a function of angular scale remarkably follows theoretical expectations, which successfully confirms the gravitational instability scenario, even on small scales where non-linear structures dominate the lensing signal.

Further evidence for lensing is provided by the gravitational magnification bias. In addition to distortion, distant objects are magnified or demagnified, depending on whether the matter along their lines-of-sight is over- or underdense compared to the average mass density. Magnified sources are preferentially included into flux-limited samples, thus sources behind matter overdensities are somewhat over-represented. Since galaxies are biased with respect to the dark-matter distribution, it

Send offprint requests to: B. Ménard,

e-mail: menard@mpa-garching.mpg.de is expected that this effect induces cross-correlations between distant sources and foreground galaxies. The existence of significant cross-correlations between distant quasars and foreground galaxies on angular scales of several arcminutes has indeed been firmly established (see Bartelmann \& Schneider 2001 for a review) and motivated further theoretical development in order to predict how the magnification bias depends on cosmological models. Following earlier work by Bartelmann (1995) and Dolag \& Bartelmann (1997), Ménard \& Bartelmann (2002) demonstrated the high sensitivity of angular quasargalaxy cross-correlation function to several cosmological parameters, namely the matter density parameter, $\Omega_{0}$, the normalisation and shape of the dark-matter power spectrum, $\sigma_{8}$ and $\Gamma$, and the bias parameter of the galaxies, $b$. Hence, magnification bias of quasars is equally efficient as cosmic shear in constraining the geometry and the dark matter power spectrum of the Universe. However, as with cosmic shear, the information provided by the quasar-galaxy correlation function alone is insufficient for independently constraining all these parameters.

Following similar motivations as Bernardeau et al. (1997) and Jain \& Seljak (1997), we decided to explore how deviations from Gaussian statistics produced by non-linear growth of structures could modify the galaxy-quasar cross-correlation signal and eventually break some degeneracies between cosmological parameters. The easiest approach is to focus on the additional information that can be extracted from higherorder correlations between quasars and galaxies which are most 
sensitive to non-Gaussianity, namely the correlation between distant quasars and foreground galaxy pairs. As for the skewness of the convergence field, we can expect that some parameter dependencies disappear by normalising the three- with twopoint correlations.

The paper is structured as follows. We briefly present the formalism of the quasar-galaxy correlation function in Sect. 2, assuming that the paradigm of gravitational instability of a Gaussian random field is valid. In Sect. 3, we then introduce the quasar-galaxy-galaxy correlator and predict some useful observational signatures. Section 4 deals with density statistics and the numerical evaluation of the triple correlator. We then define a skewness parameter in Sect. 5 and demonstrate how it can be used for directly measuring $\Omega_{0}$. Similarly, we show in Sect. 5 how several properties of the galaxy bias can be constrained. We finally estimate the signal-to-noise ratio of the corresponding observation, and apply it to the Sloan Digital Sky Survey in Sect. 6.

\section{Magnification-induced correlation functions}

Basic statistical properties of the magnification due to gravitational lensing by large-scale structure have been studied in earlier papers. To the lowest order in the relevant quantities, magnification is proportional to the lensing convergence, which has identical statistical properties to the lensing-induced distortions, i.e. the cosmic shear. Those were investigated in many studies, starting with the pioneering papers of Gunn (1967) and Blandford et al. (1992).

The two-point correlation function caused by gravitational magnification between background quasars and foreground galaxies was first introduced by Bartelmann (1995) and generalised by Dolag \& Bartelmann (1997). We refer the reader to these papers for details and only briefly recall here the formalism and approximations leading to the two-point correlation function. The notation and the definitions used for the threepoint correlation function are presented in the next section.

\subsection{The two-point correlation function $w(\theta)$}

The angular two-point correlation function between quasars and galaxies at locations $\boldsymbol{\phi}$ and $\boldsymbol{\theta}$, is defined by

$w_{\mathrm{QG}}(\theta)=\frac{\left\langle\left[n_{\mathrm{Q}}(\boldsymbol{\phi})-\bar{n}_{\mathrm{Q}}\right]\left[n_{\mathrm{G}}(\boldsymbol{\theta}+\boldsymbol{\phi})-\bar{n}_{\mathrm{G}}\right]\right\rangle}{\bar{n}_{\mathrm{Q}} \bar{n}_{\mathrm{G}}}$,

where $n_{\mathrm{Q}, \mathrm{G}}$ are the number densities of quasars and galaxies on the sky. The bar denotes the average on the sky, and the angular brackets denote averaging over positions $\phi$ and the directions of $\boldsymbol{\theta}$, assuming isotropy.

Lensing magnification increases the flux received from sources behind matter overdensities, but also stretches the sky and thus dilutes the sources, modifying their number density in the opposite direction. The net effect, an increase or a decrease of the source number density, is called the magnification bias. It depends on the number of sources gained per solid angle by the flux magnification. Let $\alpha$ be the logarithmic slope of the source number counts as a function of flux, then the number-density fluctuation is

$\frac{n_{\mathrm{Q}}(\boldsymbol{\theta})-\left\langle n_{\mathrm{Q}}\right\rangle}{\left\langle n_{\mathrm{Q}}\right\rangle}=\delta \mu^{\alpha-1}(\boldsymbol{\theta})$,

where $\delta \mu(\boldsymbol{\theta})=\mu(\boldsymbol{\theta})-1$ is the magnification fluctuation as a function of position $\boldsymbol{\theta}$. The magnification is related to convergence $\kappa$ and (the complex) shear $\gamma$ by

$\mu=\left[(1-\kappa)^{2}-|\gamma|^{2}\right]^{-1}$.

Thus, to first order in $\kappa$ and $\gamma$, the magnification fluctuation is

$\delta \mu \equiv \mu-1=2 \kappa$.

Assuming that galaxies are linearly biased with respect to the dark-matter distribution, we can write

$\frac{n_{\mathrm{G}}(\boldsymbol{\theta})-\left\langle n_{\mathrm{G}}\right\rangle}{\left\langle n_{\mathrm{G}}\right\rangle}=\bar{\delta}_{\mathrm{G}}(\boldsymbol{\theta})=\bar{b} \bar{\delta}(\boldsymbol{\theta})$,

where $\bar{b}$ is the averaged bias factor of all galaxies considered, and $\bar{\delta}$ is the projection

$\bar{\delta}(\boldsymbol{\theta})=\int_{0}^{w_{\mathrm{H}}} \mathrm{d} w p_{\delta}(w) \delta\left[f_{K}(w) \boldsymbol{\theta}, w\right]$,

of the density contrast $\delta$ along the line-of-sight, weighted by the normalised distance distribution $p_{\delta}(w)$ of the observed galaxies which are cross-correlated with the quasars. Here, $w$ is the comoving radial distance along the line-of-sight, $f_{K}(w)$ is the comoving angular diameter distance, and the upper integration boundary $w_{\mathrm{H}}$ is the comoving radial distance to the "horizon" at $z \rightarrow \infty$ (For more sophisticated biasing models, e.g. including stochasticity or non-linearity, see Pen 1998; Dekel \& Lahav 1999; Somerville et al. 2001).

We can thus write the two-point correlation function introduced in Eq. (1) as

$w_{\mathrm{QG}}(\boldsymbol{\theta})=\bar{b}\left\langle\mu^{\alpha-1}(\boldsymbol{\phi}) \bar{\delta}(\boldsymbol{\phi}+\boldsymbol{\theta})\right\rangle$.

First-order Taylor expansion of the magnification around unity yields

$w_{\mathrm{QG}}(\boldsymbol{\theta})=2 \bar{b}(\alpha-1)\langle\kappa(\boldsymbol{\phi}) \bar{\delta}(\boldsymbol{\phi}+\boldsymbol{\theta})\rangle$.

Like $\bar{\delta}, \kappa$ is a weighted projection of the density contrast along the line-of-sight. Specifically, we can write $\kappa$ as

$\kappa(\boldsymbol{\theta})=\int_{0}^{w_{\mathrm{H}}} \mathrm{d} w p_{\kappa}(w) \delta\left[f_{K}(w) \boldsymbol{\theta}, w\right]$,

where $p_{\kappa}(w)$ is the projector

$$
\begin{aligned}
p_{\kappa}(w)= & \frac{3}{2} \Omega_{0}\left(\frac{H_{0}}{c}\right)^{2} \\
& \times \int_{w}^{w_{\mathrm{H}}} \frac{\mathrm{d} w^{\prime}}{a\left(w^{\prime}\right)} W_{\mathrm{Q}}\left(w^{\prime}\right) \frac{f_{K}(w) f_{K}\left(w-w^{\prime}\right)}{f_{K}\left(w^{\prime}\right)} .
\end{aligned}
$$

The ratio between the angular diameter distances $f_{K}$ is the usual effective lensing distance, $W_{\mathrm{Q}}(w)$ is the normalised distance distribution of the quasars (see Dolag \& Bartelmann 1997 for some examples), and $a(w)$ is the cosmological scale factor. 
The correlation function $w_{\kappa \delta}$ appearing on the right-hand side of Eq. (8) can now be related to the power spectrum of the density contrast $\delta$,

$$
\begin{aligned}
w_{\kappa \delta}(\theta)= & \langle\kappa(\boldsymbol{\phi}) \bar{\delta}(\boldsymbol{\phi}+\boldsymbol{\theta})\rangle \\
= & \int_{\times\left\langle\delta\left[f_{K}(w) \boldsymbol{\theta}, w\right] \delta\left[f_{K}\left(w^{\prime}\right)(\boldsymbol{\phi}+\boldsymbol{\theta}), w^{\prime}\right]\right\rangle,} \mathrm{d} w p_{\kappa}(w) \int \mathrm{d} w^{\prime} p_{\delta}\left(w^{\prime}\right)
\end{aligned}
$$

where the integrations over $w$ and $w^{\prime}$ range from 0 to $w_{\mathrm{H}}$.

We can now use Limber's equation for the statistics of projected homogeneous and isotropic random fields. Inserting the Fourier transform of the density contrast, and introducing its power spectrum $P_{\delta}(k)$, we find

$$
\begin{aligned}
w_{\kappa \delta}(\theta)= & \int \mathrm{d} w \frac{p_{\kappa}(w) p_{\delta}(w)}{f_{K}^{2}(w)} \\
& \times \int \frac{s \mathrm{~d} s}{2 \pi} P_{\delta}\left(\frac{s}{f_{K}(w)}, w\right) J_{0}(s \theta) .
\end{aligned}
$$

\section{The three-point correlation function}

\subsection{Formalism}

Let us now extend the formalism introduced in the previous section to define higher-order statistical quantities. There are two possibilities for defining a three-point correlator between quasars and galaxies, either through correlations between single quasars and galaxy pairs, $z_{\mathrm{QGG}}$, or between quasar pairs and single galaxies, $z_{\mathrm{QQG}}$.

By definition, three-point correlations vanish for Gaussian random fields. They are created in the course of the nonlinear evolution of the underlying density field, hence they are expected to appear preferentially on small angular scales. Since the mean separation between quasars is in general much larger than between galaxies, correlations between quasars and galaxy pairs should be much easier to measure. We will therefore focus on the triple correlator $z_{\mathrm{QGG}}$ only.

By definition, and using the formalism introduced in the previous section, we have

$z_{\mathrm{QGG}}\left(\boldsymbol{\theta}_{1}, \boldsymbol{\theta}_{2}\right)=\left\langle\delta \mu^{\alpha-1}(\boldsymbol{\phi}) \bar{\delta}_{\mathrm{G}}\left(\boldsymbol{\phi}+\boldsymbol{\theta}_{1}\right) \bar{\delta}_{\mathrm{G}}\left(\boldsymbol{\phi}+\boldsymbol{\theta}_{2}\right)\right\rangle$,

where the average extends over all positions $\phi$.

Assuming as before a linear biasing relation between the galaxies and the density fluctuations, and expanding the Eq. (13) to first order in $\kappa$ and $\gamma$ leads to

$z_{\mathrm{QGG}}\left(\theta_{1}, \theta_{2}\right)=2 \bar{b}^{2}(\alpha-1) z\left(\boldsymbol{\theta}_{1}, \boldsymbol{\theta}_{2}\right)$,

where $z\left(\boldsymbol{\theta}_{1}, \boldsymbol{\theta}_{2}\right)$ is the three-point correlation function

$$
\begin{aligned}
z\left(\boldsymbol{\theta}_{1}, \boldsymbol{\theta}_{2}\right)= & \left\langle\kappa(\boldsymbol{\phi}) \bar{\delta}\left(\boldsymbol{\phi}+\boldsymbol{\theta}_{1}\right) \bar{\delta}\left(\boldsymbol{\phi}+\boldsymbol{\theta}_{2}\right)\right\rangle \\
= & \int \mathrm{d} w_{1} p_{\kappa}\left(w_{1}\right) \int \mathrm{d} w_{2} p_{\delta}\left(w_{2}\right) \int \mathrm{d} w_{3} p_{\delta}\left(w_{3}\right) \\
& \times\left\langle\delta\left[f_{K}\left(w_{1}\right) \boldsymbol{\phi}, w_{1}\right] \delta\left[f_{K}\left(w_{2}\right)\left(\boldsymbol{\phi}+\boldsymbol{\theta}_{1}\right), w_{2}\right]\right. \\
& \left.\times \delta\left[f_{K}\left(w_{3}\right)\left(\boldsymbol{\phi}+\boldsymbol{\theta}_{2}\right), w_{3}\right]\right\rangle .
\end{aligned}
$$

Again, the integrations over $w_{1,2,3}$ extend from 0 to $w_{\mathrm{H}}$. Stochastic or non-linear biasing schemes will be discussed in Sect. 5. This quantity might allow comparisons with $z_{Q \gamma \gamma}$, where $w_{\gamma \gamma}$ is the shear correlation function of some distant galaxies. Comparing the two quantities could allow to constrain the bias in an independent way.

The three-point correlation function $z_{\mathrm{QGG}}\left(\boldsymbol{\theta}_{1}, \boldsymbol{\theta}_{2}\right)$ is related to the excess probability with respect to a random distribution for finding triangle configurations, defined by the two angular separation vectors $\boldsymbol{\theta}_{1}$ and $\boldsymbol{\theta}_{2}$, formed by one quasar and two galaxies. As shown by Ménard \& Bartelmann (2002), the lensing-induced quasar-galaxy cross-correlation function $w_{\mathrm{QG}}$ has a small amplitude, typically on the order of a few per cent at angular scales of a few arcminutes. Therefore, in order to achieve a significant signal-to-noise ratio for a higher-order correlation function such as $z_{\mathrm{QGG}}$, a very large number of objects will be necessary. It is clear that the amplitude of the threepoint function depends on the specific triangle configurations. To establish an observational strategy, it would also be necessary to estimate the signal-to-noise ratios of these configurations, but we do not address this point in the present study. For our purpose of measuring a skewness parameter (see Sect. 5), the detailed angular dependence of a given triangle configuration is not of immediate relevance.

Thus, we choose to focus on an angular average of $z_{\mathrm{QGG}}\left(\boldsymbol{\theta}_{1}, \boldsymbol{\theta}_{2}\right)$ over all suitable triangle configurations inside a given aperture, which allows a measurement around each quasar. This point will be detailed in the next section, where we will also show how the averaged three-point correlation function can be observed.

\subsection{Observational signature}

The correlator $z_{\mathrm{QGG}}\left(\boldsymbol{\theta}_{1}, \boldsymbol{\theta}_{2}\right)$ can be related to the expression for the rms fluctuations of the counts of galaxies around a given quasar. Following Fry \& Peebles (1980), and considering projected (rather than three-dimensional) correlation functions, the variance of the galaxy counts in cells of solid angle $\mathcal{S}$ at fixed distance from the quasars can be written

$$
\begin{aligned}
\left\langle N^{2}\right\rangle-\langle N\rangle^{2}= & \langle N\rangle+n_{\mathrm{G}}^{2} \int_{\mathcal{S}} \mathrm{d}^{2} \boldsymbol{\theta} \mathrm{d}^{2} \boldsymbol{\theta}^{\prime}\left[w_{\mathrm{GG}}\left(\boldsymbol{\theta}-\boldsymbol{\theta}^{\prime}\right)\right. \\
& \left.+z_{\mathrm{QGG}}\left(\boldsymbol{\theta}, \boldsymbol{\theta}^{\prime}\right)-w_{\mathrm{QG}}(\boldsymbol{\theta}) w_{\mathrm{QG}}\left(\boldsymbol{\theta}^{\prime}\right)\right],
\end{aligned}
$$

where the angular brackets denote averages over ensembles of cells of size $\mathcal{S}$. Thus, we have $\langle N\rangle=n_{\mathrm{G}} \mathcal{S}+n_{\mathrm{G}} \int_{\mathcal{S}} \mathrm{d}^{2} \boldsymbol{\theta} w_{\mathrm{QG}}(\boldsymbol{\theta})$, where $n_{\mathrm{G}}$ is the galaxy number density on the sky.

If the cells were randomly placed rather than at a fixed position relative to a quasar, the variance of the galaxy counts would be

$\left\langle N^{2}\right\rangle_{\mathrm{r}}-\langle N\rangle_{\mathrm{r}}^{2}=\langle N\rangle_{\mathrm{r}}+n_{\mathrm{G}}^{2} \int_{\mathcal{S}} \mathrm{d}^{2} \boldsymbol{\theta} \mathrm{d}^{2} \boldsymbol{\theta}^{\prime} w_{\mathrm{GG}}\left(\boldsymbol{\theta}^{\prime}-\boldsymbol{\theta}\right)$,

with $\langle N\rangle_{\mathrm{r}}=n_{\mathrm{G}} \mathcal{S}$.

From Eqs. (16) and (17) we can then introduce an observable quantity $\Delta(\theta)$, defined by an extra variance of galaxy counts in cells of area $\mathcal{S}$ near quasars, normalised by $\langle N\rangle_{\mathrm{r}}^{2}$ :

$$
\begin{aligned}
\Delta(\theta) & \equiv \frac{\left\langle N^{2}\right\rangle-\langle N\rangle^{2}}{\langle N\rangle_{\mathrm{r}}^{2}}-\frac{\left\langle N^{2}\right\rangle_{\mathrm{r}}-\langle N\rangle_{\mathrm{r}}^{2}}{\langle N\rangle_{\mathrm{r}}^{2}} \\
& =\bar{z}_{\mathrm{QGG}}(\theta)-\bar{w}_{\mathrm{QG}}^{2}(\theta)+\frac{\bar{w}_{\mathrm{QG}}(\theta)}{\langle N\rangle_{\mathrm{r}}},
\end{aligned}
$$


where $\bar{w}_{\mathrm{QG}}$ and $\bar{z}_{\mathrm{QGG}}$ are the cell-averaged correlation functions

$\bar{w}_{\mathrm{QG}}(\theta)=\frac{\int \mathrm{d}^{2} \boldsymbol{\theta}^{\prime} U_{\theta}\left(\boldsymbol{\theta}^{\prime}\right) w_{\mathrm{QG}}\left(\boldsymbol{\theta}^{\prime}\right)}{\int \mathrm{d}^{2} \boldsymbol{\theta}^{\prime} U_{\theta}\left(\boldsymbol{\theta}^{\prime}\right)}$

and

$\bar{z}_{\mathrm{QGG}}(\theta)=\frac{\int \mathrm{d}^{2} \boldsymbol{\theta}_{1} U_{\theta}\left(\boldsymbol{\theta}_{1}\right) \int \mathrm{d}^{2} \boldsymbol{\theta}_{2} U_{\theta}\left(\boldsymbol{\theta}_{2}\right) z_{\mathrm{QGG}}\left(\boldsymbol{\theta}_{1}, \boldsymbol{\theta}_{2}\right)}{\int \mathrm{d}^{2} \boldsymbol{\theta}_{1} U_{\theta}\left(\boldsymbol{\theta}_{1}\right) \int \mathrm{d}^{2} \boldsymbol{\theta}_{2} U_{\theta}\left(\boldsymbol{\theta}_{2}\right)}$.

Here, the function $U_{\theta}\left(\theta^{\prime}\right)$ is a top-hat filter of radius $\theta$. Other filters can be used if one wishes to be more sensitive to specific wavelength ranges of the dark-matter power spectrum (see Schneider et al. 1998) but we will not investigate this point in the present paper.

In summary, the relevant third-order quantity for our purpose is the cell average $\bar{z}_{\mathrm{QGG}}(\theta)$ of the triple correlator $z_{\mathrm{QGG}}\left(\boldsymbol{\theta}_{1}, \boldsymbol{\theta}_{2}\right)$ between quasars and galaxy pairs. We will now estimate its properties and then show how second and thirdorder quasar-galaxy correlations can be combined to measure $\Omega_{0}$ and several properties of the galaxy bias.

\section{Evaluation of the excess galaxy variance $\Delta$}

\subsection{Statistics of the density field}

Under the common assumption that the initial density fluctuations were Gaussian and that cosmic structure grew by gravitational instability, the three-point correlation function is intrinsically a second-order quantity, and should be detectable only where non-linearities arise in the density field.

We expand the density field to second order as

$\delta(\boldsymbol{x})=\delta^{(1)}(\boldsymbol{x})+\delta^{(2)}(\boldsymbol{x})$

where $\delta^{(2)}$ is of order $\left(\delta^{(1)}\right)^{2}$ and represents departures from Gaussian behaviour. Note that since two of the three $\delta$ factors in Eq. (15) are derived from the fluctuations in the galaxy number density, this expansion is only valid if galaxies are linearly biased relative to the dark-matter fluctuations. For now we will assume this linearity and show in Sect. 5 how cosmic magnification can be used to measure the matter density parameter. The linearity of biasing has been measured on scales between 5 and $30 h^{-1} \mathrm{Mpc}$ with the $2 \mathrm{dF}$ Galaxy Redshift Survey (Verde et al. 2002). In next section, we will show that quasargalaxy correlations can also test this property and probe the angular range where this simple relation between dark matter and galaxies breaks down.

Assuming linear biasing and expanding $\delta$ to second order, we obtain for $\left\langle\delta_{1} \delta_{2} \delta_{3}\right\rangle$

$$
\begin{aligned}
\left\langle\delta_{1} \delta_{2} \delta_{3}\right\rangle & \simeq\left\langle\delta_{1}^{(1)} \delta_{2}^{(1)} \delta_{3}^{(1)}\right\rangle+\left\langle\delta_{1}^{(1)} \delta_{2}^{(1)} \delta_{3}^{(2)}\right\rangle \\
& + \text { cyclic terms }(231,312)
\end{aligned}
$$

The first term in Eq. (22) vanishes because the density fluctuation field is Gaussian to first order, hence the third moment of $\delta^{(1)}$ is zero. Thus, the leading term in Eq. (22) is of the order of $\left\langle\delta_{1}^{(1)} \delta_{2}^{(1)} \delta_{3}^{(2)}\right\rangle$.
In second-order perturbation theory, the Fourier decomposition of the density-fluctuation field is given by (Peebles 1980)

$$
\begin{aligned}
\delta^{(2)}(\boldsymbol{k})= & \int \mathrm{d}^{3} \boldsymbol{k}_{\mathbf{1}} d^{3} \boldsymbol{k}_{\mathbf{2}} \delta^{(1)}\left(k_{1}\right) \delta^{(1)}\left(k_{2}\right) \\
& \times \delta_{\mathrm{D}}\left(\boldsymbol{k}_{\mathbf{1}}+\boldsymbol{k}_{\mathbf{2}}-\boldsymbol{k}\right) F\left(\boldsymbol{k}_{\mathbf{1}}, \boldsymbol{k}_{\mathbf{2}}\right),
\end{aligned}
$$

with

$F\left(\boldsymbol{k}_{\mathbf{1}}, \boldsymbol{k}_{\mathbf{2}}\right)=\frac{5}{7}+\frac{1}{2} \frac{\boldsymbol{k}_{\mathbf{1}} \cdot \boldsymbol{k}_{\mathbf{2}}}{k_{1} k_{2}}\left(\frac{k_{1}}{k_{2}}+\frac{k_{2}}{k_{1}}\right)+\frac{2}{7} \frac{\left(\boldsymbol{k}_{\mathbf{1}} \cdot \boldsymbol{k}_{\mathbf{2}}\right)^{2}}{k_{1}^{2} k_{2}^{2}}$.

The coefficients appearing in this expression are given for an Einstein-de-Sitter universe. However, as shown by Bouchet et al. (1992), the dependences on $\Omega_{0}$ and $\Omega_{\Lambda}$ are so weak (less than $1 \%$ ) that they can be safely neglected.

Note that we have expanded the lensing-induced magnification to first order in Eq. (15). The next-order term has a contribution proportional to $\kappa^{2}$ which, when introduced in Eq. (22), is of order $\left(\delta^{(1)}\right)^{4}$ and thus formally of the same order as the other terms in Eq. (22). However, this additional term differs by the weight factor $f_{K}\left(w-w^{\prime}\right) / f_{K}\left(w^{\prime}\right)$ from the other terms since it contains the lensing efficiency. As a result, this additional factor will be one order of magnitude smaller, even though it is of the same order in the perturbation series for $\delta$. This means that the lens-lens coupling gives less contribution than the gravitational non-linear effects. (see van Waerbeke et al. 2001 for more detail). We can therefore neglect its contribution.

\subsection{Bispectrum and non-linear evolution}

The ensemble average in Eq. (22) is related to the bispectrum in Fourier space. By definition,

$$
\begin{aligned}
\left\langle\delta\left(\boldsymbol{k}_{\mathbf{1}}\right) \delta\left(\boldsymbol{k}_{\mathbf{2}}\right) \delta\left(\boldsymbol{k}_{\mathbf{3}}\right)\right\rangle= & (2 \pi)^{3} B\left(\boldsymbol{k}_{\mathbf{1}}, \boldsymbol{k}_{\mathbf{2}}, \boldsymbol{k}_{\mathbf{3}}\right) \\
& \times \delta_{\mathrm{D}}\left(\boldsymbol{k}_{\mathbf{1}}+\boldsymbol{k}_{\mathbf{2}}+\boldsymbol{k}_{\mathbf{3}}\right) .
\end{aligned}
$$

Inserting Eq. (24) into Eq. (25) leads to an expression of the bispectrum in terms of the second-order kernel $F\left(\boldsymbol{k}_{1}, \boldsymbol{k}_{2}\right)$ and the dark-matter power spectrum

$$
\begin{aligned}
B\left(\boldsymbol{k}_{1}, \boldsymbol{k}_{2}, \boldsymbol{k}_{3}\right)= & 2 F\left(\boldsymbol{k}_{1}, \boldsymbol{k}_{2}\right) P\left(k_{1}\right) P\left(k_{2}\right) \\
& +2 F\left(\boldsymbol{k}_{2}, \boldsymbol{k}_{3}\right) P\left(k_{2}\right) P\left(k_{3}\right) \\
& +2 F\left(\boldsymbol{k}_{1}, \boldsymbol{k}_{3}\right) P\left(k_{1}\right) P\left(k_{3}\right) .
\end{aligned}
$$

Note that Eq. (25) implies that the bispectrum is non-zero only if the wave vectors $\left(\boldsymbol{k}_{1}, \boldsymbol{k}_{2}, \boldsymbol{k}_{3}\right)$ form closed triangles.

For describing the bispectrum on all angular scales, we use the fitting formula for the non-linear evolution of the bispectrum derived from numerical CDM models by Scoccimarro \& Couchman (2001), extending earlier work assuming scale-free initial conditions. The kernel $F\left(\boldsymbol{k}_{1}, \boldsymbol{k}_{2}\right)$ in Eq. (26) is then simply replaced by an effective kernel $F^{\mathrm{eff}}\left(\boldsymbol{k}_{1}, \boldsymbol{k}_{2}\right)$, reading

$$
\begin{aligned}
F^{\mathrm{eff}}\left(\boldsymbol{k}_{1}, \boldsymbol{k}_{2}\right)= & \frac{5}{7} a\left(n, k_{1}\right) a\left(n, k_{2}\right) \\
& +\frac{1}{2} \frac{\boldsymbol{k}_{1} \cdot \boldsymbol{k}_{2}}{k_{1} k_{2}}\left(\frac{k_{1}}{k_{2}}+\frac{k_{2}}{k_{1}}\right) b\left(n, k_{1}\right) b\left(n, k_{2}\right) \\
& +\frac{2}{7}\left(\frac{\boldsymbol{k}_{1} \cdot \boldsymbol{k}_{2}}{k_{1} k_{2}}\right)^{2} c\left(n, k_{1}\right) c\left(n, k_{2}\right) .
\end{aligned}
$$


The coefficients $a, b$ and $c$ are given by

$$
\begin{aligned}
& a(n, k)=\frac{1+\sigma_{8}^{-0.2}(z)\left[0.7 Q_{3}(n)\right]^{1 / 2}(q / 4)^{n+3.5}}{1+(q / 4)^{n+3.5}} \\
& b(n, k)=\frac{1+0.4(n+3) q^{n+3}}{1+q^{n+3.5}} \\
& c(n, k)=\frac{1+4.5 /\left[1.5+(n+3)^{4}\right](2 q)^{n+3}}{1+(2 q)^{n+3.5}}
\end{aligned}
$$

where $n=\mathrm{d} \log P_{\operatorname{lin}}(k) / \mathrm{d} \log k$ is the local slope of the darkmatter power spectrum at wave number $k, q \equiv k / k_{\mathrm{NL}}(z)$, and $k_{\mathrm{NL}}$ is the nonlinear wave number defined by $4 \pi k_{\mathrm{NL}}^{3} P_{\text {lin }}\left(k_{\mathrm{NL}}\right)=$ 1. To be specific, $P_{\operatorname{lin}}(k)$ is the linear power spectrum at the required redshift. Finally, the function $Q_{3}(n)$ is given by

$Q_{3}(n)=\frac{\left(4-2^{n}\right)}{\left(1+2^{n+1}\right)}$,

which is the so-called saturation value obtained in "hyperextended" perturbation theory (HEPT; Scoccimarro \& Frieman 1999). These expressions imply that at large scales, where the coefficients $a, b$ and $c$ approach unity, the tree-level perturbation theory is recovered. On the other hand, at small scales, where $a^{2} \rightarrow(7 / 10) Q_{3}(n) \sigma_{8}^{-0.4}, b \rightarrow 0$ and $c \rightarrow 0$, the bispectrum becomes hierarchical with an amplitude which approximately reproduces HEPT for $\sigma_{8} \approx 1$. For more detail, see Scoccimarro \& Couchman (2001). Note that this fitting formula is accurate to $15 \%$.

\subsection{Evaluation of $\bar{z}_{\mathrm{QGG}}(\theta)$}

The formalism is now in place for computing the triple correlator $z\left(\boldsymbol{\theta}_{1}, \boldsymbol{\theta}_{2}\right)$. We start from Eq. (15) and replace the density contrast $\delta$ by its Fourier transform. Next, we employ the approximation underlying Limber's equation, which asserts that the coherence length of the density fluctuation field is much smaller than the scales on which both projectors $p_{\kappa}$ and $p_{\delta}$ vary appreciably. Finally, we insert the expression for the bispectrum described in the previous section and find

$$
\begin{aligned}
z\left(\boldsymbol{\theta}_{1}, \boldsymbol{\theta}_{2}\right)= & \int \mathrm{d} w p_{\kappa}(w) p_{\delta}^{2}(w) \int \frac{\mathrm{d}^{2} \boldsymbol{k}_{1}}{(2 \pi)^{2}} \mathrm{e}^{\mathrm{i} \boldsymbol{k}_{1} \cdot \boldsymbol{\theta}_{1} f_{K}(w)} \\
& \times \int \frac{\mathrm{d}^{2} \boldsymbol{k}_{2}}{(2 \pi)^{2}} B_{\delta}\left(k_{1}, k_{2},-\boldsymbol{k}_{1}-\boldsymbol{k}_{2}, w\right) \mathrm{e}^{\mathrm{i} \boldsymbol{k}_{2} \cdot \boldsymbol{\theta}_{2} f_{K}(w)} .
\end{aligned}
$$

We saw in Sect. 3 that the suitable quantity for observations is not directly $z\left(\boldsymbol{\theta}_{1}, \boldsymbol{\theta}_{2}\right)$, but the corresponding cell-averaged triple correlator $\bar{z}(\theta)$ defined by Eq. (20). The angular integration yields

$$
\begin{aligned}
\bar{z}(\theta)= & 4 \int \mathrm{d} w p_{\kappa}(w) p_{\delta}^{2}(w) \int \frac{\mathrm{d}^{2} \boldsymbol{k}_{1}}{(2 \pi)^{2}} \frac{J_{1}\left[k_{1} \theta f_{K}(w)\right]}{k_{1} \theta f_{K}(w)} \\
& \times \int \frac{\mathrm{d}^{2} \boldsymbol{k}_{2}}{(2 \pi)^{2}} B_{\delta}\left(k_{1}, k_{2},-\boldsymbol{k}_{1}-\boldsymbol{k}_{2}, w\right) \frac{J_{1}\left[k_{2} \theta f_{K}(w)\right]}{k_{2} \theta f_{K}(w)} .
\end{aligned}
$$

The observational signature of cosmic magnification is expressed in terms of the normalised excess scatter $\Delta(\theta)$ of galaxies around quasars; see Eq. (18). This quantity involves both the two- and three-point cell-averaged correlation functions
$\bar{w}_{\mathrm{QG}}(\theta)$ and $\bar{z}_{\mathrm{QGG}}(\theta)$. For numerically evaluating these expressions, we use the description of the non-linear evolution of the CDM power spectrum provided by Peacock \& Dodds (1996) fitting formula. We normalise the power spectrum such that the local abundance of galaxy clusters is reproduced, $\sigma_{8}=$ $0.52 \Omega_{0}^{-0.52+0.13 \Omega_{0}}$, as determined by Eke et al. (1996).

We assume for simplicity that all quasars are at the same redshift $z_{\mathrm{s}}=1.5$. More realistic quasar redshift distributions do not significantly change the following results as long as the foreground galaxies are at comparatively low redshift. We approximate the redshift distribution of the galaxies by

$p_{\delta}(z) \mathrm{d} z=\frac{\beta z^{2}}{z_{0}^{3} \Gamma(3 / \beta)} \exp \left[-\left(\frac{z}{z_{0}}\right)^{\beta}\right] \mathrm{d} z$

with $\beta=1.5$ and $z_{0}=0.3$.

The slope of the quasar number counts is fairly well constrained by the most recent quasar catalogues. We use the value $\alpha=2$ suggested by the first SDSS quasar catalogue (Schneider et al. 2001) for quasars brighter than 19th magnitude. Finally, we assume $\bar{b}=1$ for simplicity.

The left panel of Fig. 1 shows $\bar{z}_{\mathrm{QGG}}(\theta)$ (solid line) and $\bar{w}_{\mathrm{QG}}(\theta)$ (dashed line) as a function of angular scale. These quantities were computed for a low-density, spatially flat universe $\left(\Omega_{0}=0.3, \Omega_{\Lambda}=0.7\right)$. The dotted lines show the expected amplitude of $\bar{w}_{\mathrm{QG}}(\theta)$ if only linear growth of density perturbations is taken into account, and the amplitude of $\bar{z}_{\mathrm{QGG}}(\theta)$ for quasi-linear evolution. The difference between the two regimes changes the amplitude of $\bar{z}_{\mathrm{QGG}}$ by approximately two orders of magnitude on small angular scales. On large scales, the amplitude of $\bar{z}_{\mathrm{QGG}}(\theta)$ decreases quickly with $\theta$ since the density field tends to gaussianity as the smoothing scale increases, and thus the triple correlator vanishes.

Interestingly, the amplitude of $\bar{z}_{\mathrm{QGG}}$ is of the order of one per cent on arcminute scales. As for the two-point quasargalaxy correlation function, the amplitude and the shape of $\bar{z}_{\mathrm{QGG}}(\theta)$ are very sensitive to cosmological parameters.

In the right panel of Fig. 1, we plot the measurable quantity $\Delta(\theta)$ which represents the normalised excess scatter of galaxies around quasars; cf. Eq. (18). Again, we have used the $\Lambda$ CDM cosmological model, and we assume a galaxy number density of $1 \operatorname{arcmin}^{-2}$. Evidently, $\bar{z}_{\mathrm{QGG}}(\theta)$ is the main contribution to $\Delta(\theta)$ on intermediate and large angular scales. Below a few arcminutes, the term $\bar{w}_{\mathrm{QG}}(\theta) /\langle N\rangle_{\mathrm{r}}$ becomes non-negligible. This contribution is due to the shot noise of the galaxies, thus this term can be lowered when using galaxies with a higher number density.

\section{Determining $\Omega_{0}$ and testing the linearity of the bias}

The second- and third-order statistics can be used jointly so that several parameter dependences can cancel out. The underlying physical concept is that second-order statistics quantify the Gaussian characteristics of a random process, while third-order statistics are non-Gaussian contributions. When used together, one can in principle measure their relative strength, thus isolating those parameters which are most responsible for deviations from Gaussianity. 

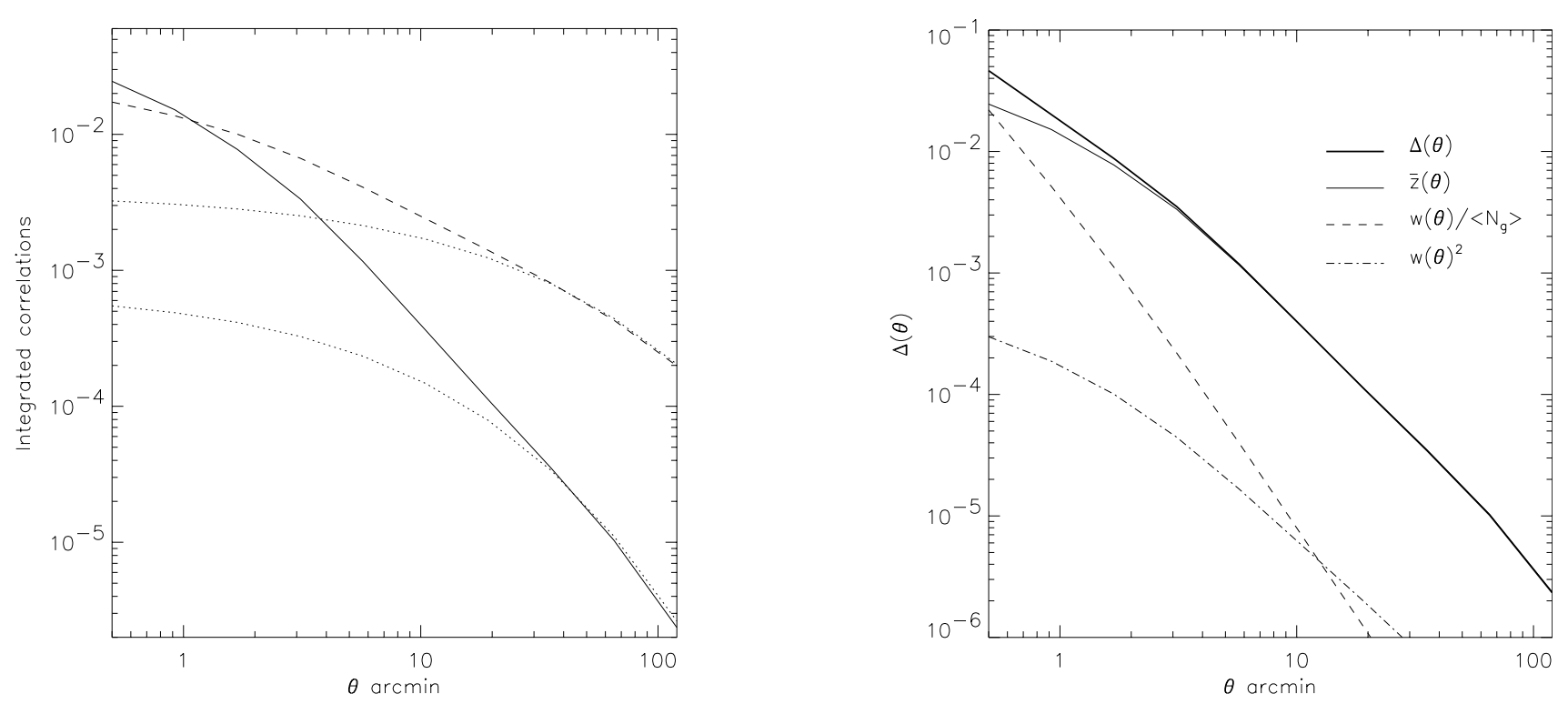

Fig. 1. Left panel: The two- and three-point quasar-galaxy correlation functions, averaged within disks of radius $\theta$, are plotted as a function of angular scale for a flat Universe with $\Omega_{0}=0.3$. All the quasars are located at redshift 1.5, and the galaxies are distributed according to Eq. (31), with a peak at $z=0.3$. The dashed curve shows the two-point correlation function $\bar{w}(\theta)$ and the solid line the three-point function $\bar{z}(\theta)$. As dotted lines are plotted the corresponding correlation functions for a linear evolution of the perturbation. Near one arcminute, the triple correlator $\bar{z}$ reaches the $\sim 2 \%$ level, assuming a linear bias of unity. At larger angular scales, the amplitude drops steeply. Right panel: We plot the expected extra scatter of galaxies around quasars for a flat Universe with $\Omega_{0}=0.3$ (thick line) and considering a galaxy density of 1 per arcmin ${ }^{2}$. We also show the different contributions to Eq. (18).

The reduced skewness (i.e. the ratio of the third and second moments of a distribution) is a useful practical estimate of non-Gaussian features in galaxy catalogs. However, in the case of cosmic magnification, it is not possible to define the skewness in the same way as for cosmic shear with the convergence field (Bernardeau et al. 1997), since we are not considering the autocorrelation properties of a single field, but the crosscorrelations between two different fields, namely the distributions of foreground galaxies and of the lensing convergence $\kappa$. Moreover, the angular-averaged three-point correlation function $\bar{z}_{\mathrm{QGG}}$ discussed in the previous section is not symmetric with respect to permutations between quasar and galaxy positions. The three-point cross-correlation of quasars with galaxy pairs involves the quasar-galaxy cross-correlation as well as the galaxy-galaxy auto-correlation. Since the latter does not contribute to the two-point quasar-galaxy correlation function, we cannot apply the usual mathematical definition of skewness which applies to a unique distribution. Instead, we define a pseudo-skewness for our purposes by the ratio

$S_{3}^{\prime}(\theta)=\frac{\bar{z}_{\mathrm{QGG}}(\theta)}{\bar{w}_{\mathrm{QG}}^{2}(\theta)}$.

Much like the skewness of cosmic shear, $S_{3}^{\prime}(\theta)$ is insensitive in the linear regime to the normalisation of the power spectrum $\sigma_{8}$, and still remains weakly dependent on $\sigma_{8}$ even in the nonlinear regime. Moreover, if the galaxy bias can be considered linear on certain angular scales and if the power spectrum is a power law, we have

$S_{3}^{\prime}(\theta)=A(\theta) \Omega_{0}^{-n}$

on such scales, where $n$ is close to one half and the amplitude $A(\theta)$ can be computed from Eqs. (12), (13) and (31).

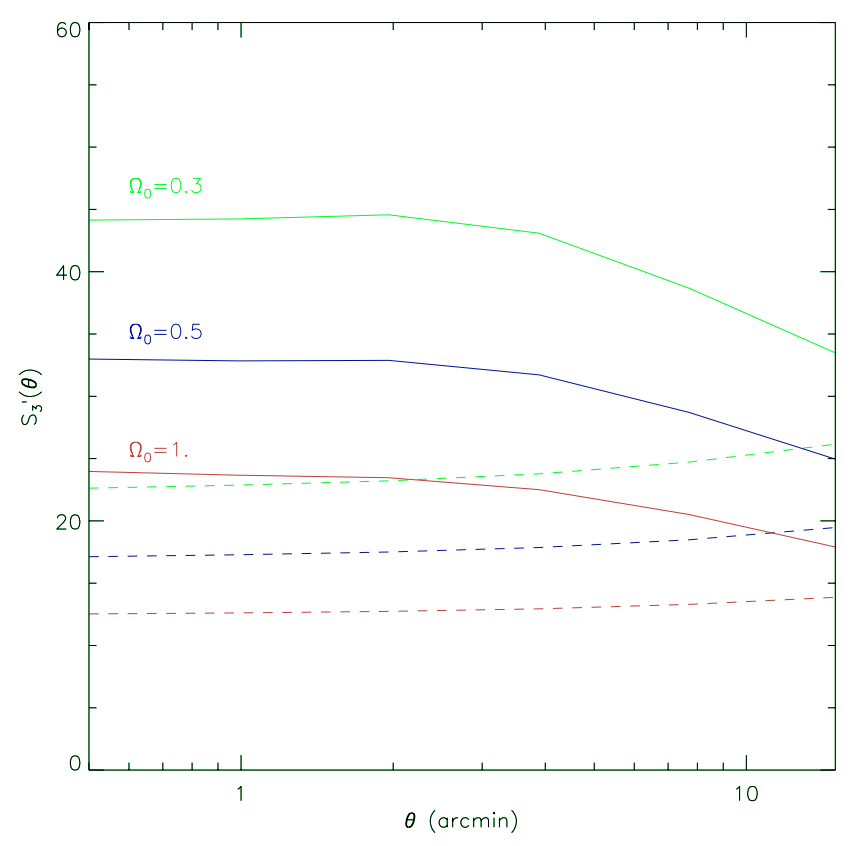

Fig. 2. The skewness $S_{3}^{\prime}(\theta)$ is shown as a function of angle $\theta$ for three different cosmological models. The solid line is the prediction based on the non-linear description of the power spectrum and the bispectrum. The dashed line shows the perturbation-theory calculation.

Interestingly, $S_{3}^{\prime}(\theta)$ does not depend on the linear galaxy bias factor. We plot the pseudo-skewness in Fig. 2 for different flat and cluster-normalised CDM model universes with density parameters $\Omega_{0}=0.3,0.5$ and 1 . The solid line shows the result obtained with the non-linear prescription of the 

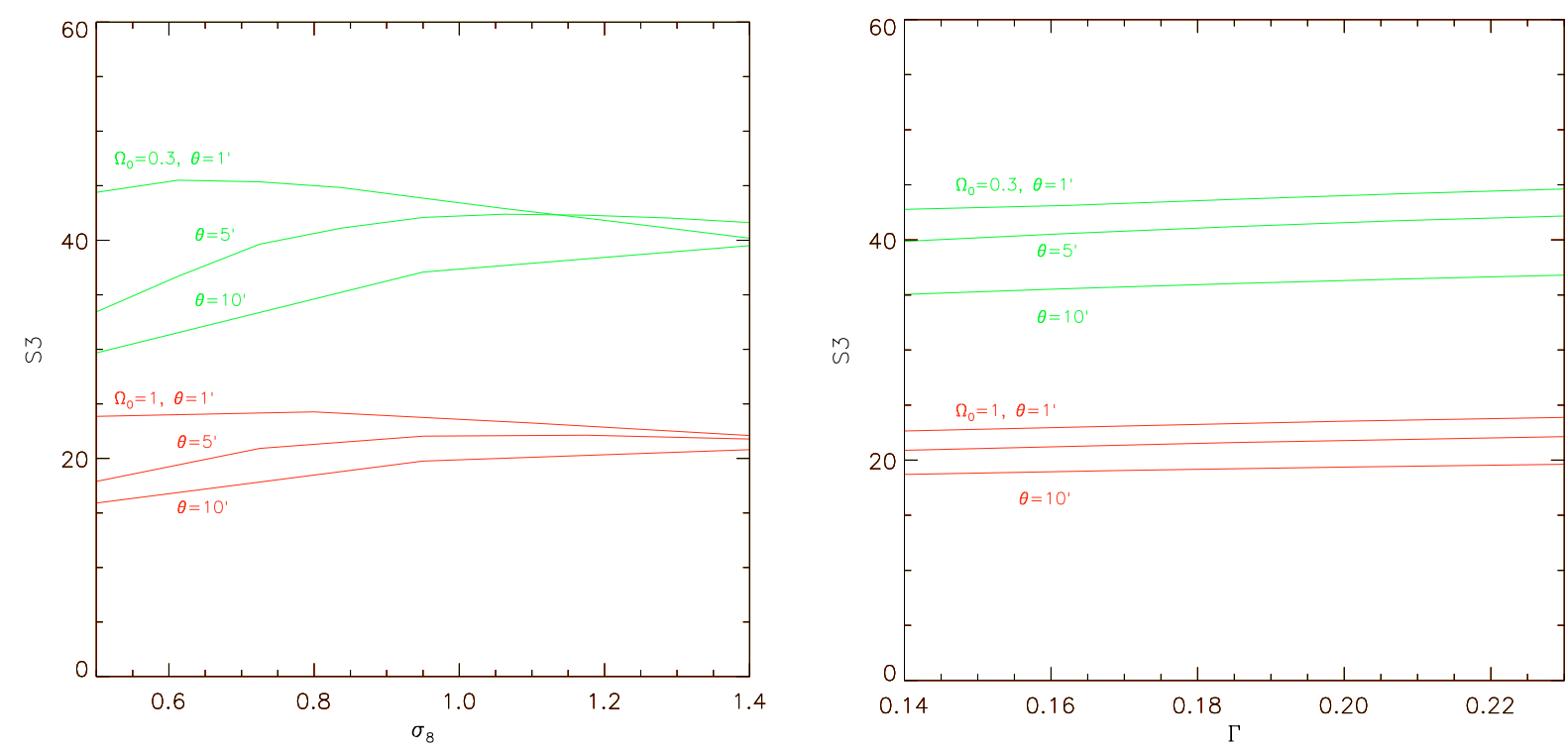

Fig. 3. The dependence of the skewness $S_{3}^{\prime}$ on $\sigma_{8}$ and $\Gamma$ is shown for two different cosmologies $\left(\Omega_{0}=1\right.$ and 0.3$)$ and three different angular scales, $1^{\prime}, 5^{\prime}$ and $10^{\prime}$. A value of $\Gamma=0.21$ is used when $\sigma_{8}$ varies and we fix $\sigma_{8}=0.9$ when $\Gamma$ varies. The dependence of $S_{3}^{\prime}$ on $\sigma_{8}$ goes down to $10 \%$ at small scales and is at the one percent level when $\Gamma$ changes.

bispectrum, and the dashed line shows the result of using quasilinear theory.

The non-linear growth of density perturbations introduces some dependency of the pseudo-skewness $S_{3}^{\prime}(\theta)$ on the normalisation $\sigma_{8}$ and the shape parameter $\Gamma$ of the dark-matter power spectrum because $\bar{w}_{\mathrm{QG}}$ and $\bar{z}_{\mathrm{QGG}}$ depend on the shape of the power spectrum in different way; cf. Eqs. (12) and (15). We plot these dependences in Fig. 3.

The figure shows that $S_{3}^{\prime}(\theta)$ depends relatively weakly on $\sigma_{8}$ and is almost insensitive to $\Gamma$. Varying $\sigma_{8}$ from 0.5 to 1.4 changes the amplitude of $S_{3}^{\prime}(\theta)$ by $30 \%$ near 10 and 5 arcminutes, and this change in the amplitude reduces to $10 \%$ near one arcminute. Thus we see that scales of a few arcminutes will be favoured for estimating $\Omega_{0}$. The effect of changing $\Gamma$ from 0.14 to 0.23 is even weaker and does not depend on the angular scale nor on the value of $\Omega_{0}$. Considering the abovementioned range, the $S_{3}^{\prime}$ dependence on $\Gamma$ is at the one percent level. Moreover, if additional measurements allow to reduce the possible ranges for $\sigma_{8}$ and $\Gamma$, the dependence of $S_{3}^{\prime}$ on these parameters is further reduced. Thus, we can consider the pseudo-skewness $S_{3}^{\prime}(\theta)$ to be weakly sensitive to normalisation of the dark-matter power spectrum, and almost insensitive to its shape parameter. Likewise, the cosmological constant $\Omega_{\Lambda}$ has a negligible effect.

The galaxy bias is expected to be linear on scales corresponding to $\sim 20^{\prime}$ and above (Verde et al. 2002). Therefore its contribution to $S_{3}^{\prime}$ cancels out in this range. Finally, the only effective parameter we are left with is $\Omega_{0}$, which means that the pseudo-skewness $S_{3}^{\prime}(\theta)$ can effectively constrain the matterdensity parameter.

The pseudo-skewness $S_{3}^{\prime}(\theta)$ can also test the linearity and stochasticity of the bias parameter which is of interest at smaller scales. For a given cosmology and assuming a CDM power spectrum, any departure from the angular variation of $S_{3}^{\prime}(\theta)$ given in Fig. 2 can be interpreted as resulting from non-linearity and/or stochasticity of the bias. Indeed, our previous calculations deriving the two- and three- point correlators used only two hypotheses, namely the linearity of the bias and the assumption that lensing effects occur in the weak regime, i.e. $\delta \mu=2 \kappa+O\left(\kappa^{2},\left|\gamma^{2}\right|\right)$. The latter relation is expected to be accurate on scales larger than a few arcminutes. Below, mediumand strong-lensing effects become non-negligible. Moreover, as described in Ménard et al. (2002), these effects can be quantified by expanding the Taylor series of the magnification to second order, thus allowing the investigation of smaller scales. Therefore, the only remaining explanation for a departure of the angular variation of $S_{3}^{\prime}(\theta)$ is a nonlinearity or stochasticity of the biasing scheme.

The pseudo-skewness can thus probe the angular range and the corresponding physical scale where the linear relation between dark matter and galaxy fluctuations breaks down.

\section{Expected signal-to-noise ratio}

We now estimate the expected signal-to-noise ratio in measurements of $\Delta(\theta)$. The determination of the effective skewness also requires a measurement of the two-point quasar-galaxy correlation function. We refer the reader to Ménard \& Bartelmann (2002) for a detailed study of the signal-to-noise ratio expected for $w_{\mathrm{QG}}(\theta)$.

A survey like SDSS will provide spectroscopic redshifts for the quasars. We can therefore safely neglect the noise coming from the uncertainty on the source redshift distribution in such a case. For the measurement of $\bar{z}_{\mathrm{QGG}}(\theta)$ we are not aiming at a detailed noise calculation, but rather an approximate estimation of the main source of error: the finite sampling error caused by the limited number of available quasars. We assume the typical size of the survey to be much larger than the angular scales we whish to probe with the correlations. As shown in Fig. 2, the dominant contribution to the excess scatter $\Delta(\theta)$ introduced in 
Eq. (18) is $\bar{z}_{\mathrm{QGG}}(\theta)$, except on small scales. We therefore use the simplifying assumption that $\Delta \sim \bar{z}_{\mathrm{QGG}}$ in the following.

Since the excess scatter of galaxies around quasars defined in Eq. (18) is a counts-in-cells estimator, its measurement accuracy will be limited by the finite size of the available sample, by boundary and edge effects, and by the effects of discrete sampling. In practice, measurements of $\bar{z}_{\mathrm{QGG}}(\theta)$ will be restricted to angular scales much smaller than the size of the survey, thus the errors contributed by boundary effects can be considered negligibly small compared to the finite sampling of the galaxies, which causes the main limitation.

In order to estimate this noise, we assume the quasars to be at random positions in the sky, i.e. uncorrelated with the galaxy positions. In fact, physical correlations are excluded given the required large separation between the two populations, and on the other hand the cross-correlations between quasars and galaxies induced by lensing are so weak that the corresponding change in the galaxy distribution is entirely negligible in the total error budget. Moreover we can neglect the quasar autocorrelations as long as the quasar mean separation is much larger than the angular scales we are interested in. With SDSS for example, this mean separation is close to $20^{\prime}$ for the sample of spectroscopic quasars satisfying $z>1$. We then assume a realistic distribution of galaxies (see Appendix A) and find the standard deviation of the normalised scatter of the galaxy counts around $N_{\text {QSO }}$ quasar positions to be

$$
\begin{aligned}
\sigma_{\theta}^{2}\left(\frac{\left\langle N^{2}\right\rangle-\langle N\rangle^{2}}{\langle N\rangle_{\mathrm{r}}^{2}}\right)= & \frac{1}{N_{\mathrm{QSO}}}\left(\frac{1}{\bar{N}^{3}}+\frac{2+3 w}{\bar{N}^{2}}+\frac{4 \bar{w}}{\bar{N}}\right. \\
& \left.+\frac{8.4 \bar{w}^{2}}{\bar{N}}+2 \bar{w}+28.2 \bar{w}^{3}\right)
\end{aligned}
$$

where $\bar{N}$ is the average number of galaxies in cells of a radius $\theta$. This result is derived in Appendix A, using the formalism developed by Szapudi \& Colombi, and considering galaxy properties measured by SDSS.

The signal we are interested in is an excess scatter in galaxy counts. Therefore, the noise of the total measurement is twice the value of $\sigma$ introduced in Eq. (34). It is possible to reduce this noise by measuring the scatter of the galaxy counts at a larger number of random locations since there are typically many more available galaxies than quasars, thus the dominant contribution comes from the measurements around quasars only. Finally, we can write the number of required quasars for achieving a $v$-signal-to-noise detection at an angular scale $\theta$ :

$N_{\mathrm{QSO}}=\frac{v^{2}}{\left[\bar{z}_{\mathrm{QGG}}(\theta) \sigma_{\theta}\right]^{2}}$,

where $v$ is the desired signal-to-noise ratio and $\sigma_{\theta}$ is the error computed from Eq. (34). We recall that the quasar number given by Eq. (35) refers to quasars with redshifts higher than those of the galaxy population.

Several weighting schemes can be used to maximise the signal-to-noise ratio of the detection. Ménard \& Bartelmann (2002) showed how to optimally weight the contribution of each quasar with respect to its magnitude. Weighting the galaxies with respect to their redshift can also increase the

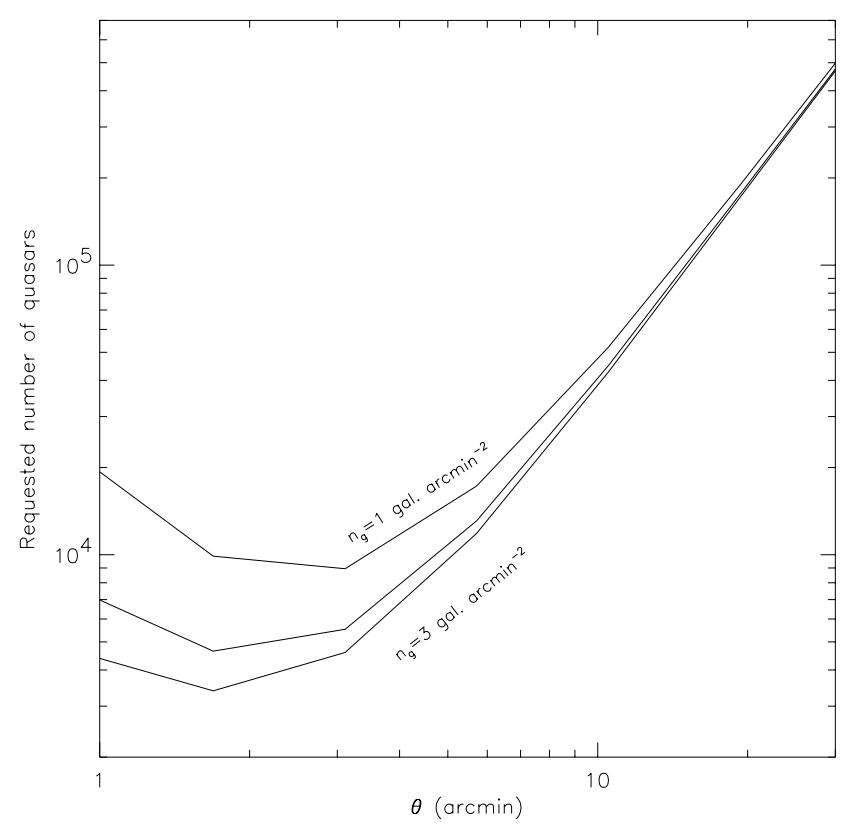

Fig. 4. Number of quasars required for a 3- $\sigma$ detection of the excess scatter $\Delta$ of the galaxy counts near quasars, as a function of angular scale $\theta$, and according to our simplified noise estimation. Three different values for the galaxy number density are assumed, namely 1,2 , and $3 \mathrm{arcmin}^{-2}$. The vertical line indicates the mean angular separation of $20^{\prime}$ between distant quasars in the SDSS.

signal-to-noise ratio of the detection. Indeed the triple correlator $z_{\mathrm{QGG}}\left(\boldsymbol{\theta}_{1}, \boldsymbol{\theta}_{2}\right)$ is related to the excess of triangle configurations in which two galaxies trace a high density region of the dark matter field, i.e. configurations in which the two galaxies are close in angle and redshift. Projection effects mimicking galaxy pairs will contribute noise to the final measurement. Therefore, the width of the galaxy redshift distribution inside a given cell around a quasar can be used to additionally weight the final measurement. Numerical simulations will be needed to quantify the change of the final signal-to-noise ratio, as well as the effects of galaxy clustering and cosmic variance which were not taken into account in our noise estimation. Moreover a precise noise estimation will also require taking into account the effects of source clustering, lens coupling and the Born approximation in the strongly non-linear regime.

As an application, we now investigate the feasibility of measuring $\Delta(\theta)$ with the data of the Sloan Digital Sky Survey (SDSS; York et al. 2000). Within this project, the sky has already been imaged for two years, and the survey will be completed in 2005, reaching a sky coverage of $\sim 10000$ square degrees. Depending on the limiting magnitude of the selected sample, SDSS can achieve a galaxy density of $\bar{n}_{\mathrm{G}} \approx 1 \mathrm{arcmin}^{-2}$ for galaxies observed down to $r^{\prime}=21$, or $\bar{n}_{\mathrm{G}} \approx 3 \mathrm{arcmin}^{-2}$ down to $r^{\prime}=22$, but requiring extensive careful masking of regions with poor seeing within the survey, and a careful stargalaxy separation (Scranton et al. 2001).

In Fig. 4, we present for different values of the galaxy number density the number of quasars required in order to achieve a 3- $\sigma$ detection of the excess scatter $\Delta(\theta)$. The figure shows that the measurement becomes more easily reachable at intermediate angular scales. Note that in our estimation we have assumed 
isolated quasars and Eq. (35) ceases to be valid at angular scales reaching the average angular separation of quasars. In the case of SDSS, this angular scale is close to $20^{\prime}$ as mentioned above. On larger angular scales, the real errors will increase due to correlated galaxy counts, and the number of quasars estimated from Eq. (35) will no longer be reliable. The SDSS will observe $10^{5}$ spectroscopic and $5 \times 10^{5}$ to $10^{6}$ photometric quasars. Thus, we conclude from Fig. 4 that SDSS will exceed the number of quasars required for measuring three-point correlations between quasars and galaxies, thus allowing a new direct measurement of the matter density $\Omega_{0}$.

\section{Conclusion}

Via the magnification bias, gravitational lensing by large-scale structures gives rise to angular cross-correlations between distant sources and foreground galaxies although the two populations are physically uncorrelated. Depending on whether the matter along the lines-of-sight towards these background sources is over- or underdense with respect to the mean, and depending on the value of the slope $\alpha$ of the cumulative number counts of the sources, magnification effects can cause an excess or a deficit of distant sources near foreground galaxies.

These lensing-induced correlations carry information on the projected dark-matter distribution along the lines-of-sight and can thus provide constraints on cosmology. Considering distant quasars and foreground galaxies, Ménard \& Bartelmann (2002) quantified these constraints and showed that given the large number of parameters involved (the matter density parameter, $\Omega_{0}$, the normalisation and shape of the darkmatter power spectrum, $\sigma_{8}$ and $\Gamma$, respectively, and the bias parameter of the galaxies, $b$ ), the information provided by the quasar-galaxy correlation function alone is insufficient for independently constraining all of these parameters.

In this paper, we have investigated what additional information can be expected from higher-order statistics. Such statistics have similar weightings of the power spectrum along the line-of-sight and can measure non-Gaussianities of the density field due to the non-linear growth of structures. We have specifically considered correlations between distant quasars and foreground galaxy pairs and showed that this three-point correlator can be related to the excess scatter of galaxies around quasars with respect to random positions, which is a straightforwardly measurable quantity. Using the assumptions that

- galaxies are linearly biased with respect to the underlying dark matter,

- the dark-matter distribution is described by a CDM power spectrum,

- and the lensing magnifications can be approximated by $\mu=$ $1+2 \kappa$ in the weak lensing regime,

we have computed the amplitude of the expected excess scatter of galaxies in the vicinity of quasars. For a flat cosmology with $\Omega_{0}=0.3$ and $\sigma_{8}=0.93$, we find the amplitude of this effect reaches the per cent level at angular scales near one arcminute.

We further showed that combining second- and third-order statistics allows a pseudo-skewness parameter $S_{3}^{\prime}$ to be defined that turns out to be weakly sensitive to the normalisation and the shape of the power spectrum. Moreover, if the linear biasing scheme is valid, this parameter is only sensitive to the matter density $\Omega_{0}$; the dependences on the other parameters $\left(\sigma_{8}, \Gamma, \Lambda\right.$ and $\bar{b}$ ) are weak or cancel out completely. Thus the skewness $S_{3}^{\prime}$ provides a direct and independent measurement of $\Omega_{0}$.

We computed the expected angular variation of $S_{3}^{\prime}$ and showed that for a given cosmology and assuming a CDM power spectrum any departure from the predicted angular shape must be due to a non-linear and/or stochastic behaviour of the galaxy bias, on scales larger than a few arcminutes.

Finally, we estimated the signal-to-noise ratio of the expected excess scatter of galaxies near quasars, which is the main source of noise in $S_{3}^{\prime}$. We derived the noise coming from the finite sampling of galaxies having realistic distributions. Applying our result to the Sloan Digital Sky Survey, we find that $S_{3}^{\prime}$ should be measurable on large scales from that survey with about twenty thousand distant and bright quasars, thus allowing a direct and independent measurement of $\Omega_{0}$.

Fewer quasars are required on smaller angular scales and the parameter $S_{3}^{\prime}$ can probe the angular range and the corresponding physical scales where the linear relation between dark matter and galaxy fluctuations may break down.

Acknowledgements. We thank Francis Bernardeau, Stéphane Colombi and Peter Schneider for helpful discussions, and Ludovic Van Waerbeke for providing his code on lensing statistics. This work was supported in part by the TMR Network "Gravitational Lensing: New Constraints on Cosmology and the Distribution of Dark Matter" of the EC under contract No. ERBFMRX-CT97-0172.

\section{Appendix A: Noise estimation}

We use the formalism developed by Szapudi \& Colombi (1996) for calculating errors due to finite sampling, and we refer the reader to this paper as well as to the review on "LargeScale Structure of the Universe and Cosmological Perturbation Theory" by Bernardeau et al. (2001). We first define some useful quantities and then derive the error on our estimator due to Poissonian noise.

We have the estimator

$E^{2}=\sigma^{2}\left(\frac{\left\langle N^{2}\right\rangle-\langle N\rangle^{2}}{\langle N\rangle^{2}}\right)=\sigma^{2}\left(\frac{\left\langle N^{2}\right\rangle}{\langle N\rangle^{2}}\right)$

Let $P_{N}$ denote the probability of finding $N$ galaxies in a cell of a given size. The factorial moments are defined as

$F_{k}=\left\langle(N)_{k}\right\rangle=\sum(N)_{k} P_{N}$

where $(N)_{k}=N(N-1) \ldots(N-k+1)$ is the $k$ th falling factorial of $N$, and the ensemble average can be evaluated using the known probability distribution $P_{N}$. We can write the error on our estimator in terms of factorial moments,

$$
\begin{aligned}
E^{2} & =\sigma^{2}\left(\frac{F_{1}+F_{2}}{F_{1}^{2}}\right) \\
& =\left\langle\left(\frac{F_{1}+F_{2}}{F_{1}^{2}}\right)^{2}\right\rangle_{C}-\left\langle\frac{F_{1}+F_{2}}{F_{1}^{2}}\right\rangle_{C}^{2},
\end{aligned}
$$


where the operator $\langle\ldots\rangle_{C}$ averages over all possible ways of throwing $C$ cells into the survey volume. In order to compute the difference, we now perturb $F_{1}$ and $F_{2}$ as $F_{1}=\bar{F}_{1}\left(1+\frac{\delta F_{1}}{\bar{F}_{1}}\right)$ and $F_{2}=\bar{F}_{2}\left(1+\frac{\delta F_{2}}{\bar{F}_{2}}\right)$, with $\left\langle\delta F_{1}\right\rangle=\left\langle\delta F_{2}\right\rangle=0$.

This yields

$$
\begin{aligned}
E^{2}= & \left\langle\delta F_{1}^{2}\right\rangle+\frac{4 \bar{F}_{2}}{\bar{F}_{1}}\left\langle\delta F_{1}^{2}\right\rangle+\frac{4 \bar{F}_{2}^{2}}{\bar{F}_{1}^{2}}\left\langle\delta F_{1}^{2}\right\rangle-\frac{2 \bar{F}_{2}^{2}}{\bar{F}_{1}^{2}}\left\langle\delta F_{1} \delta F_{2}\right\rangle \\
& -\frac{4 \bar{F}_{2}^{3}}{\bar{F}_{1}^{3}}\left\langle\delta F_{1} \delta F_{2}\right\rangle+\frac{4 \bar{F}_{2}^{4}}{\bar{F}_{1}^{4}}\left\langle\delta F_{2}^{2}\right\rangle .
\end{aligned}
$$

To evaluate this expression, we need to compute the variances and the covariance of $F_{1}$ and $F_{2}$. We will do this using the generating function of the probability distribution $P_{N}$ introduced in Eq. (A.2),

$$
P(x)=\sum_{N} P_{N} x^{N}
$$

The nice property of this quantity is that the factorial moments are obtained by a Taylor expansion of $P(x)$ around $x=1$. The cosmic covariance on factorial moments can then be written as

$\operatorname{Cov}\left(F_{i}, F_{j}\right)=\left.\left(\frac{\partial}{\partial x}\right)^{i}\left(\frac{\partial}{\partial y}\right)^{j} \mathcal{E}(x, y)\right|_{x=y=1}$,

and for estimating the finite-sampling error, the error generating function $\mathcal{E}$ is

$\mathcal{E}(x, y)=\frac{P(x y)-P(x) P(y)}{C}$

(Szapudi \& Colombi 1996), where $C$ is the number of cells used, i.e. the number of quasars or random positions involved in the measurement. Thus, we can compute the variances and covariances needed in Eq. (A.4),

$$
\begin{aligned}
\left\langle\delta F_{1}^{2}\right\rangle & =\left.\frac{1}{C} \frac{\partial}{\partial x} \frac{\partial}{\partial y}[P(x y)-P(x) P(y)]\right|_{x=y=1} \\
& =\frac{1}{C}\left[\sum_{N} N(N-1) P_{N}+N P_{N}-\left(\sum_{N} N P_{N}\right)^{2}\right] \\
& =\frac{1}{C}\left(F_{2}+F_{1}-F_{1}^{2}\right) .
\end{aligned}
$$

For Poissonian noise, we have the simple relation $F_{k}=\bar{N}^{k}$, where $\bar{N}$ is the average number of galaxies in a cell. Thus, the expression reduces to

$\left\langle\delta F_{1}^{2}\right\rangle=\frac{\bar{N}}{C}$

In the same way, we obtain

$$
\begin{aligned}
\left\langle\delta F_{2}^{2}\right\rangle & =\left.\frac{1}{C} \frac{\partial^{2}}{\partial x^{2}} \frac{\partial^{2}}{\partial y^{2}}[P(x y)-P(x) P(y)]\right|_{x=y=1} \\
& =\frac{1}{C}\left[\sum_{N} N^{2}(N-1)^{2} P_{N}-\left(\sum_{N} N(N-1) P_{N}\right)^{2}\right] \\
& =\frac{1}{C}\left(F_{4}+4 F_{3}+2 F_{2}-F_{2}^{2}\right) \\
& =\frac{4 \bar{N}^{3}+2 \bar{N}^{2}}{C}
\end{aligned}
$$

and

$$
\begin{aligned}
\left\langle\delta F_{1} \delta F_{2}\right\rangle= & \left.\frac{1}{C} \frac{\partial}{\partial x} \frac{\partial^{2}}{\partial y^{2}}[P(x y)-P(x) P(y)]\right|_{x=y=1} \\
= & \frac{1}{C}\left[\sum_{N} N^{2}(N-1) P_{N}-\sum_{N} N(N-1) P_{N}\right. \\
& \left.\times \sum_{N} N P_{N}\right] \\
= & \frac{1}{C}\left(F_{3}+2 F_{2}-F_{1} F_{2}\right) \\
= & \frac{2 \bar{N}^{2}}{C} .
\end{aligned}
$$

Inserting those terms into Eq. (A.4), we find after further simplifications

$E^{2}\left(\frac{\left\langle N^{2}\right\rangle-\langle N\rangle^{2}}{\langle N\rangle^{2}}\right)=\frac{2}{C \bar{N}^{2}}+\frac{1}{C \bar{N}^{3}}$,

which is the error due to the finite quasar number.

In order to check this result, we have performed a numerical simulation in which we measured our estimator on two-dimensional Poisson distributions of particles. We then computed its standard deviation for several cell numbers and particular point densities and find the numerical results in full agreement with the previous expression.

The clustering of galaxies can also be taken into account in this calculation. To do so, we express the factorial moments of the galaxy number counts in terms of $n$-points cell-averaged correlation functions:

$F_{k}=\bar{N}_{k}\left\langle(1+\delta)^{k}\right\rangle$.

Using the fact that $\left\langle\delta^{2}\right\rangle=\left\langle\delta^{2}\right\rangle_{\mathrm{c}}=w(\theta),\left\langle\delta^{3}\right\rangle=\left\langle\delta^{3}\right\rangle_{\mathrm{c}}=z(\theta)$ and $\left\langle\delta^{4}\right\rangle=\left\langle\delta^{4}\right\rangle_{\mathrm{c}}+3\left\langle\delta^{2}\right\rangle^{2}=w_{4}(\theta)+3 w(\theta)^{2}$ where $\bar{w}, \bar{z}$ and $\bar{w}_{4}$ are the 2-, 3- and 4-point cell-averaged correlation functions of the galaxies and the subscript $\mathrm{c}$ denotes the connected contribution, we can use the expression of the factorial moments in terms of correlation functions in the error expression (Eq. (A.4)). We find:

$$
\begin{aligned}
E^{2}\left(\frac{\left\langle N^{2}\right\rangle-\langle N\rangle^{2}}{\langle N\rangle^{2}}\right)= & \frac{1}{C \bar{N}^{3}}+\frac{2+3 \bar{w}}{C \bar{N}^{2}}+\frac{4 \bar{w}+2 \bar{z}}{C N} \\
& +\bar{w}_{4}-4 \bar{z} \bar{w}+2 \bar{w}^{2}+4 \bar{w}^{3}
\end{aligned}
$$

Under the hierarchical assumption, higher-order moments can be computed by using the skewness and kurtosis parameters of the galaxy distribution, $\bar{z}=s_{3} \bar{w}^{2}$ and $\bar{w}_{4}=s_{4} \bar{w}^{3}$. The previous expression can therefore be expressed in terms of $w(\theta)$ only. Then, each term can be evaluated by considering a power-law correlation function, $w(\theta)=\theta_{0}\left(\frac{\theta}{1 \mathrm{deg}}\right)^{-\gamma}$, and using SDSS measurements of galaxies with $20<r<21$ in order to estimate each parameter. We have $\theta_{0}=10^{-2.3}$ and $\gamma=0.7$ (Connolly et al. 2002), and for the higher-order moments of the same galaxies, $s_{3}=4.2 \pm 0.4$ and $s_{4}=41 \pm 10$ (Szapudi et al. 2002). 


\section{References}

Bacon, D., Réfrégier, A., \& Ellis, R. 2000, MNRAS, 318, 625

Bacon, D., Massey, R., Réfrégier, A., \& Ellis, R. 2002 [astro-ph/0203134]

Bartelmann, M. 1995, A\&A, 298, 661

Bartelmann, M., \& Schneider, P. 2001, Phys. Rep. 340, 291

Bernardeau, F., Colombi, S., Gaztanaga, E., \& Scoccimarro, R. 2002, Physics Reports

Bernardeau, F., van Waerbeke, L., \& Mellier, Y. 1997, A\&A, 322, 1

Bernardeau, F., Mellier, Y., \& van Waerbeke, L. 2002, A\&A, 389, L28

Bernardeau, F., van Waerbeke, L., \& Mellier, Y. 2002, A\&A, 397, 405

Blandford, R. D., \& Narayan, R. 1992, ARA\&A, 30, 311

Bouchet, F., Juszkiewicz, R., Colombi, S., \& Pellat, R. 1992, ApJ, 394, L5

Connolly, A., Scranton, R., Johnston, D., \& the SDSS collaboration 2002, ApJ, 579, 42

Dekel, A., \& Lahav, O. 1999, ApJ, 520, 24

Dolag, K., \& Bartelmann, M. 1997, MNRAS, 291, 446

Eke, V. R., Cole, S., \& Frenk, C. S. 1996, MNRAS, 282, 263

Fry, J. N., \& Peebles, P. J. E. 1980, ApJ, 238, 785

Gunn, J. E. 1967, ApJ, 150, 737

Haemmerle, H. J.-M., Miralles, J.-M., Schneider, P., et al. 2002, A\&A, 385,743

Hoekstra, H., Yee, H. K. C., Gladders, M. D., et al. 2002, ApJ, 572, 55

Jain, B., \& Seljak, U. 1997, ApJ, 484, 560

Kaiser, N., Wilson, G., \& Luppino, G. A. 2000 [astro-ph/0003338]

Maoli, R., Van Waerbeke, L., Mellier, Y., et al. 2001, A\&A, 368, 766

Ménard, B., \& Bartelmann, M. 2002, A\&A, 386, 784

Ménard, B., Hamana, T., Bartelmann, M., \& Yoshida, N. 2002, A\&A, 403,817
Peacock, J. A., \& Dodds, S. J. 1996, MNRAS, 280, L19

Peebles, P. J. E. 1980, The large scale structure of the Universe (Princeton)

Pen, U.-L. 1998, ApJ, 504, 601

Réfrégier, A., Rhodes, J., \& Groth, E. 2002, ApJ, 572, L531

Rhodes, J., Réfrégier, A., \& Groth, E. 2001, ApJ, 552, L85

Schneider, D. P., Richards, G. T., Fan, X., \& the SDSS collaboration 2002, AJ, 123, 567

Schneider, P., van Waerbeke, L., Jain, B., \& Kruse, G. 1998, MNRAS, 296, 873

Scoccimarro, R., \& Couchman, H. M. P. 2001, MNRAS, 325, 1312

Scoccimarro, R., \& Friemann, J. 1999, MNRAS, 520, 35

Scranton, R., Johnston, D., Dodelson, S., \& the SDSS Collaboration 2001, ApJ, 579, 48

Somerville, R. S., Lemson, G., Sigad, Y., et al. 2001, MNRAS, 320, 289

Szapudi, I., \& Colombi, S. 1996, ApJ, 470, 131

Szapudi, I., Frieman, J. A., Scoccimarro, R., Szalay, A. S., \& the SDSS collaboration 2002, ApJ, 570, 75

van Waerbeke, L., Mellier, Y., Erben, T., et al. 2000, A\&A, 358, 30

van Waerbeke, L., Mellier, Y., Radovich, M., et al. 2001a, A\&A, 374, 757

van Waerbeke, L., Hamana, T., Scoccimarro, R., \& Colombi, S. 2001, MNRAS, 322, 918

van Waerbeke, L., Mellier, Y., Pelló, R., et al. 2002, A\&A, 393, 369

Verde, L., Heavens, A. F., Percival, W. J., Matarrese, S., \& the 2dF collaboration 2002, MNRAS, 335, 432

Wittman, D. M., Tyson, J. A., Kirkman, D., Dell'Antonio, I., \& Bernstein, G. 2000, Nature, 405, 143

York, D. G., Adelman, J., Anderson, J. E., et al. 2000, AJ, 120, 1607 